\title{
Psychometric Evaluation of a Patient-Reported Symptom Index for Nonmuscle Invasive Bladder Cancer: Field Testing Protocol
}

Claudia Rutherford ${ }^{1}$, BPsych, PhD; Madeleine T King ${ }^{1,2}$, BSc (Hons), PhD; David P Smith ${ }^{2,3}$, MPH, PhD; Daniel SJ Costa $^{2,4}$, BSc, MPH, PhD; Margaret-Ann Tait ${ }^{1}$, BASc (Hons), MSc; Manish I Patel ${ }^{2}$, MBBS, PhD, FRACS (Urol); NMIBC-SI Working Group ${ }^{5}$

\footnotetext{
${ }^{1}$ School of Psychology, The University of Sydney, Sydney, Australia

${ }^{2}$ Sydney Medical School, The University of Sydney, Sydney, Australia

${ }^{3}$ Cancer Research Division, Cancer Council NSW, Kings Cross, Australia

${ }^{4}$ Pain Management Research Institute, Royal North Shore Hospital, St Leonards, Australia

${ }^{5}$ Nonmuscle Invasive Bladder Cancer Symptom Index Working Group, Sydney, Australia
}

\section{Corresponding Author:}

Claudia Rutherford, BPsych, PhD

The University of Sydney

School of Psychology

Level 6, Chris O'Brien Lifehouse (C39Z)

The University of Sydney

Sydney, 2006

Australia

Phone: 61286271583

Fax: 61290365292

Email: claudia.rutherford@sydney.edu.au

\section{Abstract}

Background: Nonmuscle invasive bladder cancer (NMIBC) is a chronic condition requiring intensive follow-up, repeated endoscopic examinations, tumor resections, and intravesical treatments that can occur every 3 months for life. In this clinical context, patient-reported outcomes (PROs) are a critical concern for patients and their managing clinicians. PROs have enormous potential to be integral to treatment assessment and recommendations for NMIBC; however, current PRO measures are inadequate for NMIBC because they lack key NMIBC-specific symptoms and side effects associated with contemporary treatments.

Objective: The overarching aim of this study was to develop and evaluate a patient-reported symptom index (SI) for individuals with NMIBC (the NMIBC-SI) that is acceptable to patients; reliable, valid, and responsive to differences between contemporary treatments for NMIBC; and fit for purpose as an endpoint in clinical trials.

Methods: The NMIBC-SI will be evaluated in 2 field tests across a total of 3 years. Field test 1 is a cross-sectional study design involving 225 adult NMIBC patients recruited while undergoing active treatment or those who completed final treatment within the past week. Data collected include patient demographics, clinical features of the tumor, risk category, treatment type, comorbidity, and PROs. Field test 2 is a prospective longitudinal study involving 225 newly diagnosed NMIBC-SI patients. Clinical data and patient-completed questionnaires will be collected at 4 time points during treatment: before tumor resection, 1 week after resection, end-of-induction intravesical therapy, and 1-year follow-up. Standard psychometric tests will be performed to assess the reliability, validity, responsiveness, and clinical utility of the NMIBC-SI.

Results: Participant recruitment to field test 1 commenced in February 2017. Recruitment for field test 2 is planned to commence in January 2018. Final results are expected to be published in 2019. The NMIBC-SI will be freely available for use via registration.

Conclusions: This study protocol contains detailed methods that will be used across multiple international sites. Phase 2 in the development of the NMIBC-SI will enable a comprehensive evaluation of its reliability, validity, and responsiveness to ensure that the NMIBC-SI is fit for purpose in clinical research and provides an evidence base for the ongoing improvement of future therapies for NMIBC.

Trial Registration: ClinicalTrials.gov NCT03091764; http://clinicaltrials.gov/ct2/showNCT03091764 (Archived by WebCite at http://www.webcitation.org/6umBhQeNX) 
(JMIR Res Protoc 2017;6(11):e216) doi: 10.2196/resprot.8761

\section{KEYWORDS}

quality of life; patient reported outcome measures; cancer; bladder cancer; surveys and questionnaires

\section{Introduction}

\section{Nonmuscle Invasive Bladder Cancer Is a Chronic Health Problem}

Bladder cancer is the 9th most common cancer diagnosed worldwide and the 13th most common cause of cancer death [1]. Internationally, 430,000 new cases of bladder cancer were diagnosed in 2012, with 165,000 recorded deaths [2]. In Australia alone, in 2014, an estimated 2730 people were diagnosed with bladder cancer [3], and there were 1040 recorded deaths from it [1,4]. Bladder cancer is 3 to 4 times more prevalent in males than in females, and incidence increases with age, with people older than 55 years accounting for $90 \%$ of new diagnoses [5]. Around 75\% of bladder cancer diagnoses are not muscle invasive at first diagnosis [6]. Because 5-year survival in this group exceeds $80 \%$ [7], the prevalence of nonmuscle invasive bladder cancer (NMIBC) is 10 times its incidence, even though the overall rate of recurrence is approximately $60 \%$ to $70 \%$ and progression is $20 \%$ to $30 \%$ [8].

Nonmuscle invasive bladder cancer is a chronic disease. Its management depends on the risk of the bladder cancer recurring and progressing [6]: low-risk patients receive frequent cystoscopies, possible tumor resections, and single instillations of postoperative chemotherapy; intermediate-risk patients usually require intravesical therapy, which lasts between 6 weeks and 3 years; and high-risk patients require intravesical Bacillus Calmette-Guérin (BCG; immunotherapy), which starts with 6-week induction treatment and continues with maintenance for 1 to 3 years. The higher the risk, the more the requirement for intravesical therapy. Follow-up is mandatory, with repeated endoscopic examinations, radiological imaging, biopsies, and tumor resections as frequent as 3-monthly and life-long. This makes the cost per patient from diagnosis to death the highest of all cancers [9], contributing a major economic and resource burden on health care systems [9].

Guidelines for the management of NMIBC are based on evidence for the effectiveness of treatments in reducing risk of bladder cancer progression and recurrence. Evidence for reductions in health-related quality of life (HRQoL) and associated side effects with each treatment are not incorporated into the decision-making process because they have been poorly documented (see section below on Critical Gaps in Knowledge About HRQoL and Patient-Reported Outcomes [PROs] in NMIBC). This is despite the fact that these treatments can cause substantial side effects and local and systemic toxicity. For example, the commonly used BCG has proved effective in reducing recurrences in patients with high-grade tumors and carcinoma in situ, but it can cause moderate to severe local and systemic side effects, and only $16 \%$ are able to complete their full treatment schedule [10].

\section{Why Patient-Reported Outcomes Are Important}

A PRO is any report that comes directly from patients about how they feel in relation to their health condition and its therapy, without interpretation by others [11]. PROs can include symptoms, function, HRQoL, and perceptions of treatment. These patient reports are captured and quantified by patient-reported outcome measures (PROMs) in the form of questionnaires.

PROs are beneficial for improving patient-clinician communication, prioritizing patient-centered care, and improving service provision [12,13]. In contrast to life-threatening conditions where survival endpoints may dominate HRQoL considerations, PROs should be key considerations in chronic conditions [14]. For example, a treatment found to be effective in improving survival in a clinical trial may fail in the real world because of toxicity and reduced HRQoL, which could compromise compliance and subsequently its effectiveness [15]. Similarly, the inconvenience of repeated clinic visits for monitoring or treatment can compromise compliance [16]. The acceptance of PROM-based evidence by regulatory bodies is reflected in the US Food and Drug Administration's (FDA) approval of PROs to support product labeling claims [17].

\section{Critical Gaps in Knowledge About HRQoL and PROs in NMIBC}

The primary goal in managing NMIBC in patients is to completely remove the tumor and control the unpredictable risk of recurrence and progression to muscle invasion [18] with as little treatment burden and side effects as possible. We know from clinical experience that there are many possible adverse consequences and substantial reductions in HRQoL that differ between treatment options such as chemotherapy versus BCG, induction versus maintenance therapy, and impact of single instillation chemotherapy. However, these have not been well studied or reported, and the key data on PROs are lacking for NMIBC.

The most recent indexed comprehensive review of the literature investigating the impact of NMIBC on HRQoL is over 14 years old and is based on research published between 1966 and 2000 [19]. Our recent systematic review of PROMs in NMIBC (manuscript under review; Rutherford et al) found only 6 out of 19 papers that assessed PROs used a standardized measure. Other limitations of these studies include lack of comparison groups, poor adjustment for baseline physical and psychological functions, failure to distinguish between patients with varying degrees of risk and between treatments in the analysis, and small sample sizes. Consequently, key evidence on the impact of tumor resections, repeated cystoscopies, single chemotherapy instillations, multiple chemotherapy instillations, and BCG therapies on patients' HRQoL is lacking. 
Our review did identify a NMIBC-specific PROM, the European Organisation for Research and Treatment of Cancer (EORTC)-EORTC QLQ-BLS24 questionnaire-developed in 1996. There is no publicly available information detailing its development, and it only recently underwent validation, 18 years after its development (now renamed the EORTC QLQ-NMIBC24) [20]. The validation study had some limitations: it was based on data from a single clinical trial, including only high- and intermediate-risk patients with little information about their treatments; the original content was not reviewed against contemporary treatments (which differ considerably from those in use before 1996 when the original content was developed); modifications to the scale structure were informed by clinical evidence only (ie, no patient perspective); and there is lack of assessment of test-retest reliability and clinical validity (eg, whether the module distinguishes between different risk groups for NMIBC) [20]. The question remains whether the EORTC QLQ-NMIBC24 adequately captures the impact of important symptoms and side effects associated with contemporary treatment for NMIBC.

Therefore, a patient-reported NMIBC-specific symptom index (NMIBC-SI), including possible symptoms and side effects of contemporary treatments for NMIBC, is needed to enable accurate, robust, and clinically relevant assessment of differences in PROs among contemporary treatments and to provide an evidence base for the ongoing improvement of future therapies for NMIBC. Such a measure has been developed based on existing literature and interviews with patients and clinicians [21] and pretested for face validity, comprehensiveness, comprehensibility, and clinical utility through interviews with key stakeholders (patients, urologists, and specialist nurses).

\section{Aims and Objectives}

The overarching aim of this study was to develop and evaluate the NMIBC-SI for reliability, validity, and responsiveness to ensure that it is fit for purpose in clinical research.

\section{Psychometric Aims}

- To evaluate the feasibility and acceptability of the NMIBC-SI; produce a shorter version, if appropriate, by selecting items that perform best against robust psychometric criteria; examine the legitimacy of summing items into scales; identify legitimate scales and subscales; and test scaling assumptions and scale performance in a large-scale Australian sample (field test 1).

- To psychometrically evaluate the measurement properties of the final version SI, testing for reliability, clinical validity (sensitivity to differences between patient groups and responsiveness to clinically important change), and interpretability of the final version NMIBC-SI in a new large-scale international sample (field test 2).

- To conduct a head-to-head comparison of the new NMIBC-SI with the EORTC bladder cancer module, QLQ-NMIBC24.

\section{Clinical Aims (Field Test 2)}

- To assess and compare key PROs across the full range of contemporary treatments for NMIBC and over-the-disease trajectory, including acute treatment and 1-year survivorship.

- To compare PROs between patients with low-, intermediate-, and high-risk NMIBC.

\section{Methods}

\section{Overview of Project Research Design}

This multicenter study was designed to evaluate the psychometric properties of an NMIBC-SI for patients treated for NMIBC. Guidance for developing and validating health outcome measures has been followed to ensure high quality and standardization for the development of the NMIBC-SI $[11,22,23]$. The guidance recommends that collaboration and expert discussion are sought and utilized through all stages of development, and it proposes distinct phases for the development and evaluation of PROMs. The research design includes 2 phases: (1) development of NMIBC-SI in 2 parts, conceptual framework to generate items and pretesting; and (2) evaluation of NMIBC-SI in 2 parts, a preliminary field test 1 for item reduction and a final field test 2 for psychometric properties (Figure 1). Phase 1 has been conducted, and results are summarized below.

In preliminary research and phase 1 development, a conceptual framework was developed by tapping into 3 sources: (1) a systemic review and narrative analysis of the HRQoL and PRO literature, identifying several local and systematic side effects associated with contemporary treatments for NMIBC (eg, urinary problems, discomfort, and malaise); (2) in-depth qualitative interviews with a sample of NMIBC patients that explored patients' experience of receiving treatment; and (3) in-depth qualitative interviews with treating clinicians (specialist nurses and urologists) that explored important issues from their perspective. This phase was important to ensure that high content validity was achieved and demonstrated [24]. An exhaustive list of clinically relevant issues (items) was generated from the conceptual framework and patient verbatims [21].

In phase 1 pretesting, further qualitative interviews explored the generated list of issues with patients and clinicians for clarity and overlap and the appropriateness of the NMIBC-SI's time frame, question stem, and response options. On the basis of information obtained from the interviews, the provisional NMIBC-SI was revised to produce a preliminary version ready for field testing.

The evaluation of the NMIBC-SI is phase 2 of this project. It will be undertaken in 2 parts: preliminary field test 1 (item reduction) and final field test 2 (psychometric properties). The preliminary field test will identify any items with poor psychometric performance for possible elimination. The final field test will be undertaken to evaluate the item-reduced version of the NMIBC-SI for reliability, validity, and responsiveness. Gold standard psychometric methods will be used [11,22,23].

\section{Phase 2: Field Test 1}

The psychometric properties of the NMIBC-SI will be evaluated in 2 field tests, including a preliminary field test (item reduction) to identify items with poor psychometric properties for possible elimination and to identify subscales, and a final field test 
(psychometric evaluation) to evaluate the reliability and validity of the item-reduced version of the NMIBC-SI. The overall strategy and methods for the psychometric evaluation are based on the methods used to develop and validate PROMs in several other areas of medicine and surgery [24-26].

\section{Design for Preliminary Field Test 1 (Item Reduction)}

The purpose of the preliminary field test 1 is to produce a short (item-reduced) version of the NMIBC-SI and to undertake an initial psychometric evaluation of the item-reduced questionnaire. This will be done using a cross-sectional study design.

An item reduction strategy developed for evaluation of PROMs in other medical areas [25-27] will be used to: (1) identify items on the provisional version of the NMIBC-SI with poor psychometric properties for possible elimination; (2) conduct a preliminary evaluation of NMIBC-SI subscales; and (3) undertake a preliminary evaluation of the acceptability, reliability, and validity of the item-reduced NMIBC-SI.

Figure 1. Development and evaluation of the symptom index for individuals with nonmuscle invasive bladder cancer.

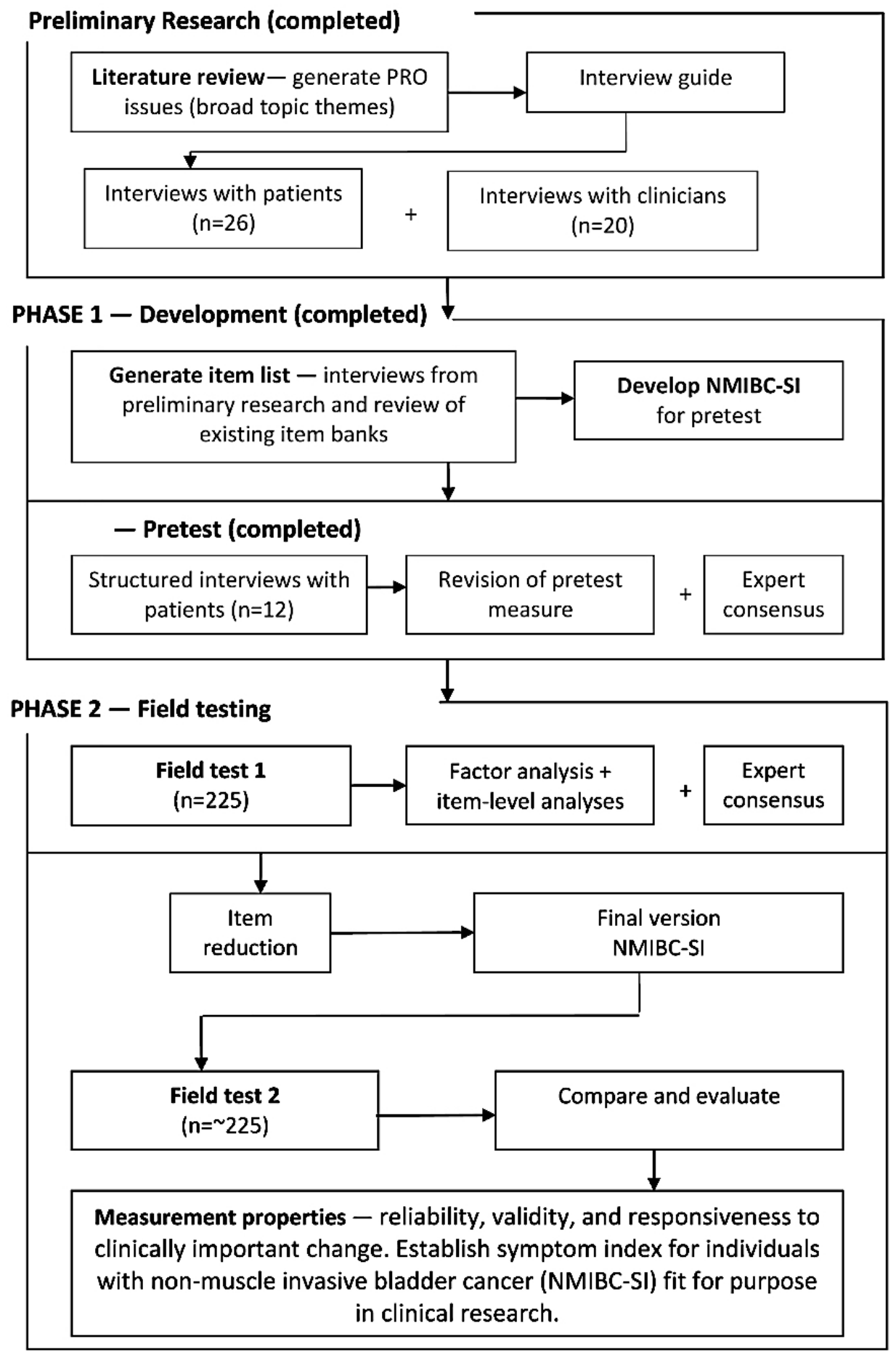


Results of the item reduction analyses will be used to develop a shorter version of NMIBC-SI for final psychometric field testing.

\section{Eligibility}

\section{Inclusion Criteria}

Adult patients (aged $\geq 18$ years) from participating centers diagnosed with NMIBC, who are able to read and understand English and give their written informed consent will be included in the study. Patients will be recruited while undergoing active treatment (ie, 1 week after tumor resection or intravesical therapy) or when they have completed final treatment for NMIBC within the past week.

\section{Exclusion Criteria}

Patients will be excluded from the study if any of the following criteria apply:

- They are unconscious or confused.

- They have cognitive impairment.

- They are unable to speak, read, and/or write in English.

- They are diagnosed with muscle invasive disease.

- They are unable to provide informed consent.

\section{Sample Size}

Approximately 225 NMIBC patients will be required, purposively sampled to ensure representation of patients across the 3 NMIBC risk categories (Textbox 1) [6]. This sample size is based on recommendations for psychometric analyses of new summated scales; 5 to 10 subjects per item are needed to reduce the chance effect of sampling [28,29]. Following this recommendation, a 30-item NMIBC-SI would require a sample between 150 and 300 patients.

\section{Recruitment and Consent Procedures}

Patients from participating centers who meet the eligibility criteria will be informed about and invited to take part in the study either in person or via an invitation letter. Consecutive patients will be identified and approached to participate by either the named site investigator or the named site nurse. Accrual will be reviewed to ensure that there is balanced representation of patients in all NMIBC categories. A record of those identified as eligible, approached to participate, refusals, consenting patients, and questionnaire returns will be kept for progress monitoring and final reporting purposes (see Data Collection/Assessment section below).

A verbal explanation of the study and a patient information sheet will be provided for patients to consider. These will include detailed information about the rationale, design, and personal implications of the study. Following information provision, patients will have as much time as they need to consider participation. The right of the patient to refuse consent without giving reasons will be respected.

Assenting patients will then be invited to provide informed, written consent on the consent form at the end of the patient information sheet to collect baseline assessment data and to complete the questionnaire. The patient will remain free to withdraw from the study at any time without giving reasons and without prejudicing any further treatment. The original consent form will be filed within the investigator site file or at a designated secure location.

\section{Registration}

Following informed consent and confirmation of eligibility, participants will be provided with a study ID number and registered to the study.

\section{Screening}

The participating site staff will complete a log of all patients screened for eligibility. Screening logs will be returned to the University of Sydney.

Using the study ID number as an identifier, information will be collected for all eligible patients, including the age, gender, risk category, and treatment type.

For those who decline to participate in the study, reasons will be recorded.

Textbox 1. Nonmuscle invasive bladder cancer (NMIBC) patient risk groups.

\author{
High-risk tumors \\ Any of the following: \\ - $\quad$ T1 tumor \\ - G3 (high grade, which is a mixture of some G2 and G3) tumor \\ - Carcinoma in situ (CIS) \\ Intermediate-risk tumors \\ - All tumors not defined in the adjacent categories (between the category of low- and high risk) \\ Low-risk tumors \\ - Primary, solitary, Ta, G1 (PUNLMP, low grade is a mixture of G1 and G2), $<3 \mathrm{~cm}$, no CIS
}

- Multiple and recurrent and large ( $>3 \mathrm{~cm}$ ) Ta G1G2 tumors (all conditions must be presented at this point); low grade is a mixture of G1 and G2 


\section{Data Collection/Assessment}

Study data will be recorded by participating site staff on case report forms and by participants on questionnaire booklets either on paper or electronically. The study data are collected and managed using REDCap electronic data capture tools hosted at the University of Sydney [30].

Assessments will be undertaken as follows:

- Registration and baseline data

- Patient questionnaire booklet

Registration and clinical data will be completed by the clinician or specialist nurse at participating centers. Hard-copy questionnaires will be collected by the clinician or specialist nurse or posted by the patient back to the site. Study ID number coded data will be sent to the University of Sydney for central data management. Individually identifiable data and master lists linking study ID numbers to individual identity will be retained by the participating sites.

\section{Registration and Clinical Data}

Patients who meet the inclusion criteria and provide informed consent will be registered to this study. Registration and clinical information will be recorded by participating site staff including the following:

- $\quad$ Patient study ID number

- Age

- Gender

- Country of birth

- Marital status

- Living arrangements

- Education

- Risk category (clinical definitive category from medical records)

- Tumor grade

- Tumor stage

- Treatment type (information about which treatment interventions the patient is currently receiving)

- Comorbidity (Charlson Comorbidity Index [31] and history of prostate cancer)

- Name of site staff member completing clinical data

- Confirmation of eligibility

\section{Questionnaire Booklet}

Participants will self-complete the questionnaire booklet containing the NMIBC-SI, which will be provided to them by the clinician or specialist nurse at participating centers in hard copy or by link to the Web-based version. It is anticipated that completion of the questionnaire may take up to $20 \mathrm{~min}$. Completed hardcopy questionnaires will be entered into REDCap by participating site staff directly or returned to the University of Sydney for data entry.

\section{Mode of Questionnaire Administration}

Participants will be given a hard copy of the questionnaire or provided with a link to the Web-based questionnaire, depending on their preference.

\section{Analyses and Statistical Considerations}

To determine whether the NMIBC-SI fulfills fundamental prerequisites for rigorous measurement as defined by established psychometric criteria [32] and the US FDA guidance [11], standard psychometric tests for acceptability, data quality, internal reliability, and factor analysis construct validity will be performed. Table 1, adapted from Gorecki, 2013 [33], presents full details of the tests and criteria used in the psychometric evaluation.

The purpose of the item reduction analysis is to produce a psychometrically robust version of the NMIBC-SI. Standard psychometric tests and criteria, as described in Table 1, will be performed to identify and retain items with strong psychometric properties and eliminate items with poor psychometric properties to produce a shorter, item-reduced version of the NMIBC-SI. These analyses will also evaluate the hypothesized subscales of the NMIBC-SI.

Missing data will not be imputed. The frequency of missing data will be determined, and items with a response rate of $<80.0 \%(180 / 225)$ will be investigated.

\section{Phase 2: Field Test 2-Evaluation of Reliability and Clinical Validity}

\section{Design for Field Test 2}

The purpose of the final field test 2 is to assess the reliability and clinical validity (sensitivity to differences between patient groups and responsiveness to clinically important change) and interpretability of the final version NMIBC-SI in a new large-scale international sample. This phase will be conducted using a prospective longitudinal study design. The outcome will be a patient-reported NMIBC-SI that is reliable and clinically valid, with NMIBC-specific content that complements the more generic HRQoL content of the EORTC QLQ-C30.

\section{Eligibility}

Adult patients (aged $\geq 18$ years) from participating centers diagnosed with NMIBC, after imaging or flexible cystoscopy but before endoscopic resection, who are able to read and understand English and give their written informed consent will be included in the study.

\section{Sample Size}

Approximately 225 patients newly diagnosed with NMIBC will be required to provide sufficient subjects for statistical assessment of test-retest reliability [34,35] and clinical validity in terms of both sensitivity to differences between patient groups [36] and responsiveness to clinically important change [37]. See section on Sample Size for Field Test 1 for sample size justification. 
Table 1. Psychometric tests and criteria.

\begin{tabular}{lll}
\hline Property & Definition/test & Criteria for acceptability \\
\hline 1. Item analysis & $\begin{array}{l}\text { Identify items for possible elimination due to weak } \\
\text { psychometric performance; assessed on the basis } \\
\text { of (1) exploratory factor analysis with principal } \\
\text { axis factoring and (2) item- and scale-level analy- }\end{array}$ & $\begin{array}{l}\text { Exploratory factor analysis: items with a factor- } \\
\text { loading coefficient } \geq 0.4 \text { will be retained in each } \\
\text { subscale. }\end{array}$ \\
& $\begin{array}{ll}\text { Applied to all items: (1) missing data }<5 \% ; ~(2) \\
\text { maximum endorsement frequencies }<80 \% \text { (ie, the } \\
\text { proportion of respondents who endorse each re- } \\
\text { sponse category), including floor or ceiling effects } \\
& <80 \% \text { (ie, response categories with high endorse- } \\
& \text { ment rates at the bottom and top ends of the scale, } \\
& \text { respectively); (3) evidence of item responsiveness } \\
\text { as assessed by significant improvement between } & \text { baseline and test of cure assessments (field test } 2 \\
\text { only) }\end{array}$ \\
&
\end{tabular}

2. Acceptability

The quality of data; assessed by completeness of data and score distributions
The extent to which items comprising a scale measure the same construct (eg, homogeneity of the scale); assessed by Cronbach alpha and itemtotal correlations

3.2 Test-retest reliability (field test 2 only) The stability of a measuring instrument; assessed by administering the instrument to respondents on two different occasions and examining the correlation between test and retest scores

\section{Validity}

4.1 Content validity

\subsection{Construct validity}

Within-scale analyses

\section{Analyses against external criteria}

Convergent validity

Discriminant validity

Known groups differences

5. Responsiveness
The extent to which the content of a scale is representative of the conceptual domain it is intended to cover; assessed qualitatively during the questionnaire development stage through pretesting with patients, expert opinion, and literature review

Evidence that a single entity (construct) is being measured and that items can be combined to form a summary score

Evidence that the scale is correlated with other measures of the same or similar constructs; assessed on the basis of correlations between the measure and other similar measures

Evidence that the scale is not correlated with measures of different constructs; assessed on the basis of correlations with measures of different constructs

The ability of a scale to differentiate known groups; assessed by comparing scores for subgroups that are expected to differ on the construct being measured

The ability of a scale to detect clinically significant change following treatment of known efficacy; assessed by examining within-person change scores before and after treatment and calculating an effect size statistic (mean change score divided by standard deviation of pretreatment scores)
Qualitative evidence from patients, expert opinion, and literature review that items in the scale are representative of the construct being measured

Confirmatory factor analysis: (1) items with a factor-loading coefficient $\geq 0.4$ and (2) moderate to high correlations between scale scores

Correlations are expected to vary according to the degree of similarity between the constructs that are being measured by each instrument. Specific hypotheses are formulated and predictions tested on the basis of correlations

Low correlations between the instrument and measures of different constructs

Significant differences between known groups or difference of expected magnitude

Moderate to large effect sizes (small 0.2, moderate 0.5 , and large 0.8 or higher) (page number not for citation purposes) 
Table 2. Patient-reported outcome assessment schedule.

\begin{tabular}{|c|c|c|c|c|}
\hline Risk group & $\begin{array}{l}\text { Assessed within } 3 \text { months } \\
\text { before tumor resection }\end{array}$ & $\begin{array}{l}\text { Assessed within } 4 \text { to } 10 \text { days } \\
\text { after the tumor resection }\end{array}$ & $\begin{array}{l}\text { Assessed within } 1 \text { month } \\
\text { after the end of induction } \\
\text { intravesical therapy }^{\mathrm{a}}\end{array}$ & $\begin{array}{l}\text { Assessed within } 1 \text { month } \\
\text { before the 1-year cystoscopy } \\
\text { (or at early cessation due to } \\
\text { adverse events }^{\text {b }} \text { ) }\end{array}$ \\
\hline High & $\mathrm{T} 1$ & $\mathrm{~T} 2$ & T3 & $\mathrm{T} 4$ \\
\hline Intermediate & $\mathrm{T} 1$ & $\mathrm{~T} 2$ & $\mathrm{~T} 3$ & $\mathrm{~T} 4$ \\
\hline Low & $\mathrm{T} 1$ & $\mathrm{~T} 2$ & $\mathrm{~T} 3^{\mathrm{c}}$ & $\mathrm{T} 4$ \\
\hline$\sim \mathrm{n}$ & 225 & 225 & 225 & 225 \\
\hline
\end{tabular}

${ }^{\mathrm{a}} \mathrm{A}$ minimum of 25 participants from each risk group will be asked to complete an additional questionnaire pack 3 to 7 days after T3.

${ }^{\mathrm{b}}$ Preferably before cystoscopy.

${ }^{\mathrm{c}}$ For the low-risk group, T3 will be 8 weeks after resection.

\section{Recruitment and Consent Procedures}

Patients will be recruited at diagnosis, after imaging or flexible cystoscopy but before endoscopic resection. Eligible patients will be identified by the clinician investigators and their teams at participating centers, ensuring adequate representation of patients in the 3 risk groups (see Textbox 1) [6]. Patients who meet eligibility criteria will be approached in person and informed about and invited to take part in the study. Consent procedures are similar to field test 1 , that is, using patient information sheet and consent form for field test 2 .

\section{Data Collection/Assessment}

\section{Registration and Baseline Data}

Registration data will be collected as done for field test 1 . Baseline questionnaires will be completed before endoscopic resection (Table 2).

\section{Follow-Up Data Collection}

Participants will complete follow-up questionnaire packs at scheduled follow-up time points (see Table 2). The clinician or specialist nurse at participating centers will be responsible for sending follow-up questionnaires, emails, and reminders to participants. Participants will either be posted a hard-copy questionnaire pack or emailed a link to their follow-up questionnaires, depending on their preference. Participants who complete the Web-based questionnaire pack will be given an option to provide their email address to receive automatic reminders from the REDCap system at follow-up time points. Participants will be advised that email addresses are stored within the REDCap system and used solely for the purpose of sending reminders. REDCap is a secure Web app that runs on the University of Sydney's servers, ensuring that data stay within the Sydney University data center. The provision of an email address for reminders is entirely voluntary, and participants will be free to change their mind at any point, after which any email address provided will be removed from the REDCap system.

\section{PRO Assessment Time Points}

Prospective assessment of newly diagnosed patients before and after treatment is required for the responsiveness analysis. It is expected that the NMIBC-SI will detect changes in symptoms due to specific treatments. As risk profile determines treatment schedule, the corresponding schedules of prospective assessments are indicated in Table 2. These time points will also provide PRO data to compare between treatment and risk groups and over time, addressing our clinical aims (see Aims and Objectives section).

In addition to the planned assessments (Table 2), a minimum of 25 participants sampled from each of the 3 risk groups will complete an additional NMIBC-SI 3 to 7 days after the T3 administration to evaluate test-retest reliability. The length of the test-retest interval must be short enough to ensure that clinical change in the NMIBC status is unlikely to occur but sufficiently long to ensure that respondents do not recall their responses from the first assessment. A short test-retest interval is necessary to ensure that stability per se is being evaluated, rather than clinical change during the test-retest interval, which will underestimate the NMIBC-SI's reliability.

Participants will be sent a hard copy or link to the questionnaires 2 weeks before their scheduled follow-up time point. Up to 2 email or telephone reminders to complete follow-up questionnaires will be made if questionnaires are not completed by the follow-up date. The clinician or specialist nurse at participating centers will be responsible for sending follow-up questionnaires, emails, and reminders to participants unless participants who complete the Web-based questionnaires have provided their email address to receive automatic reminders directly from REDCap. The assessment time windows are indicated in Table 2.

\section{Mode of Questionnaire Administration}

Participants will be given a hard copy of the questionnaire or provided with a link to the Web-based questionnaire depending on their preference.

\section{Questionnaire Booklet}

A questionnaire pack containing the NMIBC-SI, EORTC QLQ-C30, and the EORTC QLQ-NMIBC24 will be self-completed by all participants via hard copy or Web.

Participants will complete the item-reduced version of the NMIBC-SI, the QLQ-C30, and the QLQ-NMIBC24 measures to assess construct validity (convergent, discriminant, and known groups; see Table 2). The guiding principle in selecting the validating measures is to include measures that will allow a comparison of NMIBC-SI subscales with measures of similar 
constructs (convergent validity) and with measures of different constructs (discriminant validity), and to compare NMIBC-SI scores in clinically defined known groups whose HRQoL would be expected to differ.

The QLQ-C30 [38] is a core questionnaire for evaluating the HRQoL of patients participating in cancer clinical trials. It is a 30 -item questionnaire with 9 multi-item subscales and 6 single items. It incorporates 5 functional scales (physical, role, cognitive, emotional, and social functioning), 3 symptom scales (fatigue, pain, and nausea or vomiting), and a global health status or HRQoL scale. The single items assess dyspnea, appetite loss, sleep disturbance, constipation, diarrhea, and perceived financial impact of disease and treatment. Ratings for each item range from 1 (not at all) to 4 (very much). The QLQ-C30 is designed to be used across cancer populations and takes about $11 \mathrm{~min}$ to complete [38].

The QLQ-NMIBC24 [20] is a 24-item questionnaire for evaluating the HRQoL of patients with superficial (nonmuscle invasive) bladder cancer. The questionnaire is designed to supplement the QLQ-C30 and incorporates 6 multi-item scales and 5 single items. Ratings for each item range from 1 (not at all) to 4 (very much). The scales cover urinary symptoms, malaise, worries about the future, bloating and flatulence, sexual function, and male sexual problems. The single items assess intravesical treatment issues, sexual intimacy, sexual enjoyment, risk of contaminating partner, and female sexual problems.

All measures will be administered in the same order. It is anticipated that completion of questionnaire packs may take up to $20 \mathrm{~min}$.

\section{Analyses and Statistical Considerations}

Analyses will evaluate the measurement properties of the final version of the NMIBC-SI, using psychometric tests described for field test 1 (Table 1). Additional tests for reliability (test-retest: correlations for summary scores $\geq .70$ ), clinical validity (in terms of sensitivity to groups known to differ clinically and responsiveness to clinically meaningful change over time [37]), and estimation of minimal important difference (interpretability) [39] will be undertaken. Evaluation of subscales will be determined by factor analysis and against external criteria (between-group validity: convergent, discriminant, and known group differences validity). To evaluate convergent and discriminant validity, we will compare NMIBC-SI scales with the scales within the EORTC QLQ-C30 and QLQ-NMIBC24 measures. NMIBC-SI scores for patients by risk and treatment groups will be compared to evaluate known group differences. Risk groups are defined as low, intermediate, and high (see Textbox 1) [6]. Treatment groups are defined as follows: (1) cystoscopy alone or TURBT, (2) course of intravesical chemotherapy, and (3) course of intravesical BCG. We hypothesize that low-risk patients will have lower levels of treatment-related problems at follow-up time points compared with high-risk patients, who have more intensive treatments. Factor analysis, together with the results of other item-level analyses described in Table 1, will be used to investigate hypothesized subscales.
Analyses of the clinical aims will include: (1) evaluation of PRO changes over time, from diagnosis to peak treatment and at 1 year; (2) comparison of PROs between the 3 risk groups at each time point; and (3) head-to-head comparison of the relative discriminatory ability of the NMIBC-SI and the EORTC QLQ-NMIBC24. The pretumor resection assessment (T1) will be at diagnosis and is considered our baseline assessment. Patients are not expected to have any treatment-related problems at this point. The subsequent 3 time points (Table 2) are intended to capture short-term, intermediate, and long-term levels of problems, and each will be compared with baseline (T1).

\section{Results}

\section{Development of NMIBC-SI}

A patient-reported NMIBC-SI, including all symptoms and side effects associated with contemporary treatments for NMIBC, has been developed based on a conceptual framework of PROs important to patients with NMIBC and their managing clinicians [21].

The conceptual framework was developed by utilizing 3 sources (see Figure 1): (1) a systematic review and narrative analysis of the PRO literature relevant to NMIBC identified several local and systemic side effects associated with contemporary treatments for NMIBC (eg, urinary problems, discomfort, and flu-like symptoms); (2) in-depth qualitative interviews with a sample of NMIBC patients explored patients' experience of receiving treatment; and (3) in-depth qualitative interviews with treating clinicians (specialist nurses and urologists) explored important issues from their perspective. These aspects were important to ensure that the new NMIBC-SI had high content validity [24]. An exhaustive list of clinically relevant issues (items) was generated from the conceptual framework and patient and clinician verbatims.

\section{Pretesting of the NMIBC-SI}

The draft version of the NMIBC-SI was pretested using structured interviews with key stakeholders (patients and clinicians), testing for face validity, relevance and comprehensiveness of content, comprehensibility, and clinical utility. Specifically, clarity and overlap of items and the appropriateness of the NMIBC-SI's time frame, question stem, and response options were explored. On the basis of information obtained from the interviews, the provisional NMIBC-SI was revised to produce a preliminary version ready for field testing.

The NMIBC-SI was designed to complement and be administered alongside the EORTC QLQ-C30 generic cancer questionnaire [38] (which includes core domains of functioning and HRQoL, fatigue and general pain but no NMIBC-specific symptoms).

\section{Item Reduction of the NMIBC-SI: Field Test 1}

The field testing phase is planned over 3 years. Recruitment for field test 1 commenced in February 2017 in 9 Australian centers. Recruitment for field test 2 is planned to commence in January 2018 in the same 9 Australian centers that participated in field test 1 plus an additional 10 international centers (2 in New Zealand, 4 in the United States, 2 in Canada, and 2 in Europe) 
following the universal approach to using the same language in different countries [40]. These 19 centers include both public and private hospitals and treatment clinics. Final results are expected to be published in 2019. The NMIBC-SI will be freely available for use via registration.

\section{Discussion}

This study protocol contains detailed methods to be used across 19 international sites, including both public and private hospitals and treatment clinics that treat patients diagnosed with NMIBC. Field test 1 is a cross-sectional study and includes a sample of patients with NMIBC on active treatment. Field test 2 is a longitudinal study and includes a sample of newly diagnosed patients to enable assessment of possible treatment effects as well as responsiveness of the NMIBC-SI. No patients recruited for field test 1 will be included in field test 2 . Phase 2 in the development of the NMIBC-SI will enable a comprehensive evaluation of its reliability, validity, and responsiveness to ensure that it is fit for purpose in clinical research and provides an evidence base for the ongoing improvement of future therapies for NMIBC.

Following evaluation, our NMIBC-SI will be suitable for use with English-speaking patients, diagnosed and treated for NMIBC in Australia, New Zealand, the United States, Canada, and the United Kingdom. Cross-cultural and language translations are planned following development and evaluation of the English version.

\section{Acknowledgments}

This study is sponsored by the University of Sydney with grant funding from Cancer Australia and Cancer Council NSW, Australia (ID: 1103036). The contents of this protocol are solely the responsibility of the authors and do not reflect the views of Cancer Australia or the Cancer Council NSW.

The authors thank the NMIBC-SI Working Group and participating site staff for their contribution to patient recruitment and the Australian and New Zealand Urogenital and Prostate (ANZUP) Cancer Trials Group for their collaboration. NMIBC-SI working group members are Dr Paul Anderson, Prof Maurizio Brausi, Dr Venu Chalasani, Prof Mark Frydenberg, Prof Dickon Hayne, A/Prof Shomik Sengupta, Mr Peter Stanford, and Dr Roger Watson.

The authors thank all the study participants.

\section{Conflicts of Interest}

None declared.

\section{Multimedia Appendix 1}

Peer Review Report.

[PDF File (Adobe PDF File), 482KB-Multimedia Appendix 1]

\section{References}

1. Cheluvappa R, Smith DP, Cerimagic S, Patel MI. A comprehensive evaluation of bladder cancer epidemiology and outcomes in Australia. Int Urol Nephrol 2014 Jul;46(7):1351-1360. [doi: 10.1007/s11255-014-0643-z] [Medline: 24488686]

2. Globocan.iarc.fr. 2014. GLOBOCAN 2012: estimated cancer incidence, mortality and prevalence worldwide in 2012 URL: http://globocan.iarc.fr/Default.aspx [accessed 2017-10-27] [WebCite Cache ID 6uVs4NpGX]

3. Australian Institute of Health and Welfare (AIHW). Webarchive.nla. 2014. Summary pages for selected cancers 2014 URL: http://webarchive.nla.gov.au/gov/20150622042034/http://www.aihw.gov.au/cancer/cancer-in-australia-overview-2014/ appendixb/ [accessed 2017-10-26] [WebCite Cache ID 6uVsMEafk]

4. Australian Institute of Health and Welfare (AIHW). Aihw. Canberra: AIHW; 2014. Australian cancer incidence and mortality (ACIM) books URL: http://www.aihw.gov.au/acim-books [WebCite Cache ID 6tpMmLpDQ]

5. Ploeg M, Aben KK, Kiemeney LA. The present and future burden of urinary bladder cancer in the world. World J Urol 2009 Jun;27(3):289-293 [FREE Full text] [doi: 10.1007/s00345-009-0383-3] [Medline: 19219610]

6. Babjuk M, Burger M, Zigeuner R, Shariat SF, van Rhijn BW, Compérat E, European Association of Urology. EAU guidelines on non-muscle-invasive urothelial carcinoma of the bladder: update 2013. Eur Urol 2013 Oct;64(4):639-653. [doi: 10.1016/j.eururo.2013.06.003] [Medline: 23827737]

7. Anastasiadis A, de Reijke TM. Best practice in the treatment of nonmuscle invasive bladder cancer. Ther Adv Urol 2012 Feb;4(1):13-32 [FREE Full text] [doi: 10.1177/1756287211431976] [Medline: 22295042]

8. Zlotta AR, van Vooren JP, Huygen K, Drowart A, Decock M, Pirson M, et al. What is the optimal regimen for BCG intravesical therapy? Are six weekly instillations necessary? Eur Urol 2000 Apr;37(4):470-477. [Medline: 10765079]

9. Botteman MF, Pashos CL, Redaelli A, Laskin B, Hauser R. The health economics of bladder cancer: a comprehensive review of the published literature. Pharmacoeconomics 2003;21(18):1315-1330. [Medline: 14750899] 
10. Lamm DL, Blumenstein BA, Crissman JD, Montie JE, Gottesman JE, Lowe BA, et al. Maintenance bacillus Calmette-Guerin immunotherapy for recurrent TA, T1 and carcinoma in situ transitional cell carcinoma of the bladder: a randomized Southwest Oncology Group Study. J Urol 2000 Apr;163(4):1124-1129. [Medline: 10737480]

11. Resources.crfhealth. 2009. Guidance for Industry: patient reported outcome measures: use in medical product development to support labeling claims URL: http://resources.crfhealth.com/regulatory-guidance/ guidance-for-industry-patient-reported-outcome-measures-use-in-medical-product-development-to-support-labeling-claims [accessed 2017-10-30] [WebCite Cache ID 6uaXTOQgn]

12. Bottomley A, Flechtner H, Efficace F, Vanvoorden V, Coens C, Therasse P, European Organisation for Research and Treatment of Cancer (EORTC) Data Center and Quality of Life Group. Health related quality of life outcomes in cancer clinical trials. Eur J Cancer 2005 Aug;41(12):1697-1709. [doi: 10.1016/j.ejca.2005.05.007] [Medline: 16043345]

13. Snyder CF, Aaronson NK, Choucair AK, Elliott TE, Greenhalgh J, Halyard MY, et al. Implementing patient-reported outcomes assessment in clinical practice: a review of the options and considerations. Qual Life Res 2012 Oct;21(8):1305-1314. [doi: 10.1007/s11136-011-0054-x] [Medline: 22048932]

14. Lipscomb J, Gotay CC, Snyder CF. Patient-reported outcomes in cancer: a review of recent research and policy initiatives. CA Cancer J Clin 2007;57(5):278-300 [FRE Full text] [doi: 10.3322/CA.57.5.278] [Medline: $\underline{17855485}$ ]

15. Metwally MA, Frederiksen KD, Overgaard J. Compliance and toxicity of the hypoxic radiosensitizer nimorazole in the treatment of patients with head and neck squamous cell carcinoma (HNSCC). Acta Oncol 2014 May;53(5):654-661. [doi: 10.3109/0284186X.2013.864050] [Medline: 24328536]

16. Borras JM, Sanchez-Hernandez A, Navarro M, Martinez M, Mendez E, Ponton JL, et al. Compliance, satisfaction, and quality of life of patients with colorectal cancer receiving home chemotherapy or outpatient treatment: a randomised controlled trial. Br Med J 2001 Apr 7;322(7290):826 [FREE Full text] [Medline: 11290635]

17. Patrick DL, Burke LB, Powers JH, Scott JA, Rock EP, Dawisha S, et al. Patient-reported outcomes to support medical product labeling claims: FDA perspective. Value Health 2007;10 Suppl 2:S125-S137 [FREE Full text] [doi: 10.1111/j.1524-4733.2007.00275.x] [Medline: 17995471]

18. Aldousari S, Kassouf W. Update on the management of non-muscle invasive bladder cancer. Can Urol Assoc J 2010 Feb;4(1):56-64 [FREE Full text] [Medline: 20165581]

19. Botteman MF, Pashos CL, Hauser RS, Laskin BL, Redaelli A. Quality of life aspects of bladder cancer: a review of the literature. Qual Life Res 2003 Sep;12(6):675-688. [Medline: 14516177]

20. Blazeby JM, Hall E, Aaronson NK, Lloyd L, Waters R, Kelly JD, et al. Validation and reliability testing of the EORTC QLQ-NMIBC24 questionnaire module to assess patient-reported outcomes in non-muscle-invasive bladder cancer. Eur Urol 2014 Dec;66(6):1148-1156 [FREE Full text] [doi: 10.1016/j.eururo.2014.02.034] [Medline: 24612661]

21. Rutherford C, Costa DS, King MT, Smith DP, Patel MI. A conceptual framework for patient-reported outcomes in non-muscle invasive bladder cancer. Support Care Cancer 2017 Apr 27;25(10):3095-3102. [doi: 10.1007/s00520-017-3717-5] [Medline: 28451912]

22. Johnson C, Aaronson N, Blazeby JM, Bottomley A, Fayers P, Koller M, et al. Groups.eortc.be. Brussels: EORTC Quality of Life Group; 2011 Apr. EORTC quality of life group guidelines for developing questionnaire modules URL: http://groups. eortc.be/qol/sites/default/files/archives/guidelines_for_developing_questionnaire-_final.pdf [accessed 2017-10-27] [WebCite Cache ID 6uW5Avvoa]

23. Streiner DL, Norman GR, Cairney J. Health Measurement Scales: A Practical Guide to Their Development and Use. Oxford: Oxford University Press; 2015.

24. Stead ML, Brown JM, Velikova G, Kaasa S, Wisløff F, Child JA, et al. Development of an EORTC questionnaire module to be used in health-related quality-of-life assessment for patients with multiple myeloma. European Organization for Research and Treatment of Cancer Study Group on Quality of Life. Br J Haematol 1999 Mar;104(3):605-611. [Medline: 10086801]

25. Lamping DL, Schroter S, Marquis P, Marrel A, Duprat-Lomon I, Sagnier PP. The community-acquired pneumonia symptom questionnaire: a new, patient-based outcome measure to evaluate symptoms in patients with community-acquired pneumonia. Chest 2002 Sep;122(3):920-929. [Medline: 12226033]

26. Smith SC, Lamping DL, Banerjee S, Harwood R, Foley B, Smith P, et al. Measurement of health-related quality of life for people with dementia: development of a new instrument (DEMQOL) and an evaluation of current methodology. Health Technol Assess 2005 Mar;9(10):1-93, iii-iv [FREE Full text] [Medline: 15774233]

27. Lamping DL, Schroter S, Kurz X, Kahn SR, Abenhaim L. Evaluation of outcomes in chronic venous disorders of the leg: development of a scientifically rigorous, patient-reported measure of symptoms and quality of life. J Vasc Surg 2003 Feb;37(2):410-419 [FREE Full text] [doi: 10.1067/mva.2003.152] [Medline: 12563215]

28. McColl E. Developing questionnaires. In: Fayers P, Hays R, editors. Assessing Quality of Life in Clinical Trials: Methods and Practice. New York: Oxford University Press; 2005.

29. Nunnally JC, Bernstein IH. Psychometric Theory. New York: McGraw-Hill; 1994.

30. Harris PA, Taylor R, Thielke R, Payne J, Gonzalez N, Conde JG. Research electronic data capture (REDCap)--a metadata-driven methodology and workflow process for providing translational research informatics support. J Biomed Inform 2009 Apr;42(2):377-381 [FREE Full text] [doi: 10.1016/j.jbi.2008.08.010] [Medline: 18929686] 
31. Charlson ME, Pompei P, Ales KL, MacKenzie CR. A new method of classifying prognostic comorbidity in longitudinal studies: development and validation. J Chronic Dis 1987;40(5):373-383. [Medline: $\underline{3558716]}$

32. Allen MJ, Yen WM. Introduction to Measurement Theory. Monterey, CA: Waveland Press; 1979.

33. Gorecki C, Brown JM, Cano S, Lamping DL, Briggs M, Coleman S, et al. Development and validation of a new patient-reported outcome measure for patients with pressure ulcers: the PU-QOL instrument. Health Qual Life Outcomes 2013 Jun 13;11:95 [FREE Full text] [doi: 10.1186/1477-7525-11-95] [Medline: 23764247]

34. Fleiss J. Reliability of measurements. In: Fleiss J, editor. The Design and Analysis of Clinical Experiments. New York: John Wiley \& Sons; Feb 1986:1-32.

35. Guyatt G, Walter S, Norman G. Measuring change over time: assessing the usefulness of evaluative instruments. J Chronic Dis 1987;40(2):171-178. [Medline: 3818871]

36. Cocks K, King MT, Velikova G, Martyn SM, Fayers PM, Brown JM. Evidence-based guidelines for determination of sample size and interpretation of the European Organisation for the Research and Treatment of Cancer Quality of Life Questionnaire Core 30. J Clin Oncol 2011 Jan 1;29(1):89-96. [doi: 10.1200/JCO.2010.28.0107] [Medline: 21098316]

37. King MT, Bell ML, Costa D, Butow P, Oh B. The Quality of Life Questionnaire Core 30 (QLQ-C30) and Functional Assessment of Cancer-General (FACT-G) differ in responsiveness, relative efficiency, and therefore required sample size. J Clin Epidemiol 2014 Jan;67(1):100-107. [doi: 10.1016/j.jclinepi.2013.02.019] [Medline: 24125895]

38. Aaronson NK, Ahmedzai S, Bergman B, Bullinger M, Cull A, Duez NJ, et al. The European Organization for Research and Treatment of Cancer QLQ-C30: a quality-of-life instrument for use in international clinical trials in oncology. J Natl Cancer Inst 1993 Mar 3;85(5):365-376. [Medline: $\underline{\text { 8433390] }}$

39. King MT. A point of minimal important difference (MID): a critique of terminology and methods. Expert Rev Pharmacoecon Outcomes Res 2011 Apr;11(2):171-184. [doi: 10.1586/erp.11.9] [Medline: 21476819]

40. Wild D, Eremenco S, Mear I, Martin M, Houchin C, Gawlicki M, et al. Multinational trials-recommendations on the translations required, approaches to using the same language in different countries, and the approaches to support pooling the data: the ISPOR Patient-Reported Outcomes Translation and Linguistic Validation Good Research Practices Task Force report. Value Health 2009 Jun;12(4):430-440 [FREE Full text] [doi: 10.1111/j.1524-4733.2008.00471.x] [Medline: 19138309]

\author{
Abbreviations \\ BCG: Bacillus Calmette-Guérin \\ EORTC: European Organisation for Research and Treatment of Cancer \\ HRQoL: health-related quality of life \\ NMIBC: nonmuscle invasive bladder cancer \\ NMIBC-SI: symptom index for individuals with NMIBC or NMIBC-specific symptom index \\ PRO: patient-reported outcome \\ PROM: patient-reported outcome measures \\ SI: symptom index
}

\author{
Edited by G Eysenbach; submitted 16.08.17; peer-reviewed by B Ansa, N Azevedo; comments to author 20.09.17; revised version \\ received 03.10.17; accepted 03.10.17; published 08.11 .17 \\ Please cite as: \\ Rutherford C, King MT, Smith DP, Costa DSJ, Tait MA, Patel MI, NMIBC-SI Working Group \\ Psychometric Evaluation of a Patient-Reported Symptom Index for Nonmuscle Invasive Bladder Cancer: Field Testing Protocol \\ JMIR Res Protoc 2017;6(11):e216 \\ URL: http://www.researchprotocols.org/2017/11/e216/ \\ doi: $10.2196 /$ resprot. 8761 \\ PMID: 29117930
}

CClaudia Rutherford, Madeleine T King, David P Smith, Daniel SJ Costa, Margaret-Ann Tait, Manish I Patel, NMIBC-SI Working Group. Originally published in JMIR Research Protocols (http://www.researchprotocols.org), 08.11.2017. This is an open-access article distributed under the terms of the Creative Commons Attribution License (https://creativecommons.org/licenses/by/4.0/), which permits unrestricted use, distribution, and reproduction in any medium, provided the original work, first published in JMIR Research Protocols, is properly cited. The complete bibliographic information, a link to the original publication on http://www.researchprotocols.org, as well as this copyright and license information must be included. 